\title{
Physical nature of intermolecular interactions inside Sir2 homolog active site: molecular dynamics and ab initio study
}

\author{
Przemysław Czeleń $^{1} \cdot$ Żaneta Czyżnikowska $^{2}$
}

Received: 15 January 2016 / Accepted: 24 April 2016 /Published online: 6 May 2016

(C) The Author(s) 2016. This article is published with open access at Springerlink.com

\begin{abstract}
In the present study, we analyze the interactions of NAD+-dependent deacetylase (Sir2 homolog yeast Hst2) with carba-nicotinamide-adenine-dinucleotide (ADP-HPD). For the Sir2 homolog, a yeast Hst2 docking procedure was applied. The structure of the protein-ADP-HPD complex obtained during the docking procedure was used as a starting point for molecular dynamics simulation. The intermolecular interaction energy partitioning was performed for proteinADP-HPD complex resulting from molecular dynamics simulation. The analysis was performed for ADP-HPD and 15 amino acids forming a deacetylase binding pocket. Although the results indicate that the first-order electrostatic interaction energy is substantial, the presence of multiple hydrogen bonds in investigated complexes can lead to significant value of induction component.
\end{abstract}

Keywords Docking procedure $\cdot$ Molecular dynamic simulations $\cdot$ Intermolecular interaction energy partitioning

\section{Introduction}

Weak noncovalent interactions involving molecules of biological importance have been identified to play an important role

Przemysław Czeleń

przemekcz@cm.umk.pl

Żaneta Czyżnikowska

zaneta.czyznikowska@gmail.com

1 Department of Physical Chemistry, Collegium Medicum, Nicolaus Copernicus University, Kurpińskiego 5, 85-950 Bydgoszcz, Poland

2 Department of Inorganic Chemistry, Wrocław Medical University, Borowska 211, 55-556 Wrocław, Poland in many fundamental processes in nature. The detailed analysis of strength of hydrogen bonds and London dispersion interactions can provide a better understanding of the structure and stability of biomolecules [1]. The forces are responsible for specificity of DNA-protein binding. Many pharmaceutical ligand-protein interactions are noncovalent in their nature. Although the X-ray measurements results are often available and give a lot of geometrical information, the magnitude and character of interaction between the substrate-specific ligand and enzymes need further exploration.

Due to their biochemical properties Sir2 (silent information regulator 2) proteins are involved in various biological processes such as DNA metabolism, regulation, and repair of double-stranded breaks $[2,3]$. This group of proteins can be involved in transcriptional silencing, apoptosis and chromosome stability. Their promoting activity in longevity in yeast mother cells was also proved [4]. It has been also indicated that some Sir2 homologs in eukaryotes have been implicated in the proper cell cycle progression, radiation resistance, and genomic stability [5]. To date, investigations strongly suggest that their biological activity is largely dependent on their deacetylate properties and is also partially modulated by concentration of nicotinamide in cells. The conformation of Sir2 is unique among all Sir proteins and was carefully determined [6]. In the current study we present the structural and energetical consequences of interactions between NAD+-dependent deacetylase (Sir2) with carba-nicotinamide-adeninedinucleotide (ADP-HPD) [7]. According to Sanders' study, nicotinamide molecule is bound to the D-pocket which has hydrophobic character due to the PHE67, PHE184, around the pyridine ring and PHE44 together with ILE117 proximal to the carboxamide moiety (See Fig. 1). The docking procedure and molecular dynamics simulations were performed in order to gain an insight into the structural and energetical basis 
Fig. 1 Graphic representation of Hst2 active site with ADP-HPD.

Marked amino acids play the most important role in creation of interactions with ligands

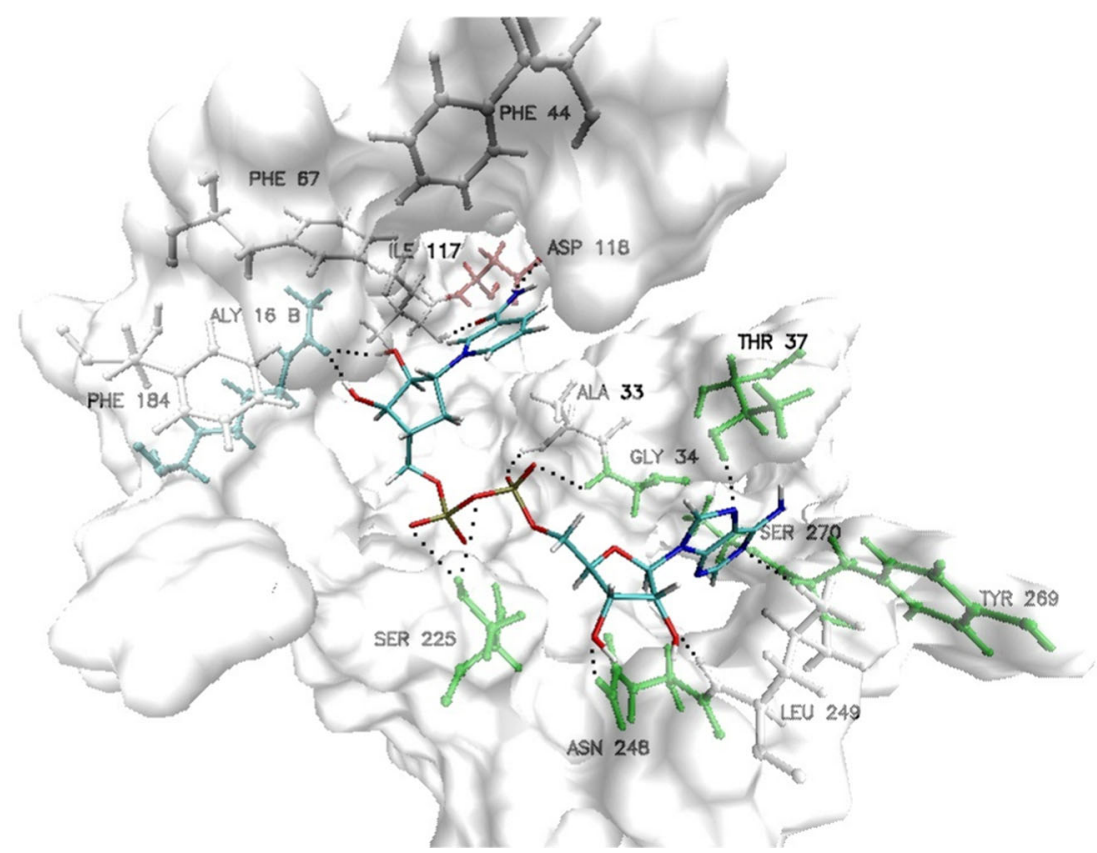

of Sir2 enzyme inhibition. Moreover, the total intermolecular energy between protein and ADP-HPD was divided into physically relevant terms determining the nature of interactions in complex in question.

\section{Methods}

\section{Docking procedure}

During the docking procedure, the structure of the Sir2 homo$\log$ with an acetyllysine histone $\mathrm{H} 4$ peptide yeast Hst2 was used. The structural data of the considered protein, denoted as 1SZC [8], was downloaded from the Protein Data Bank. Both structures used during the docking procedure, namely Sir2 protein and optimized ADP-HPD ligand, contained only polar hydrogen atoms. The docking stage was performed with use of the united-atom scoring function implemented in AutoDock Vina [9]. The space declared during the docking procedure $(18 \times 20 \times 24)$ has been limited to the active site of Sir2 enzyme. The docking procedure for considered subunits was repeated ten times with exhaustiveness equal 30; its outcomes are presented in Fig. 2. In all cases, atoms of ADP-HPD ligand backbone are localized almost in this same point of conformational space of the active site. Small differences are mainly related to localization of hydrogen atoms.

\section{Molecular dynamics simulation}

The structure of the Hst2-ADP-HPD complex obtained during docking procedure was used as a starting point for molecular dynamics simulation. Ligand structure was characterized with the use of amber force field parameters and the atomic charges were calculated according to the Merz-Kollman scheme via the RESP procedure [10] at HF/6-31G* level. The complex structure was neutralized and immersed in a periodic box of a TIP3P water box [11]. The analyzed system was heated to $300 \mathrm{~K}$ during $100 \mathrm{ps}$ of MD simulation and the temperature was controlled by a Langevin thermostat [12]. The periodic boundary conditions and SHAKE algorithm [13] were applied for $60 \mathrm{~ns}$ of molecular dynamic simulation. The analysis of molecular dynamics simulation parameters, namely potential and kinetic energies of system and RMSD values, confirm that the first $20 \mathrm{~ns}$ of obtained trajectory is an equilibration period. Values presented in Fig. 3 confirm that both elements of the considered system, namely protein and ADPHPD ligand, after this stage reached dynamic stability. The final

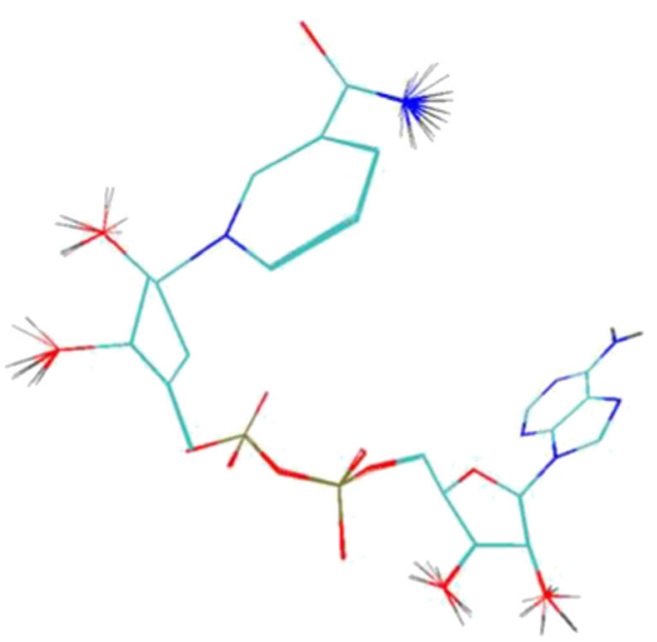

Fig. 2 ADP-HPD ligand conformations obtained during docking procedure 
Fig. 3 Distribution of RMSD values for Sir 2 protein and ADPHPD ligand during molecular dynamics simulation

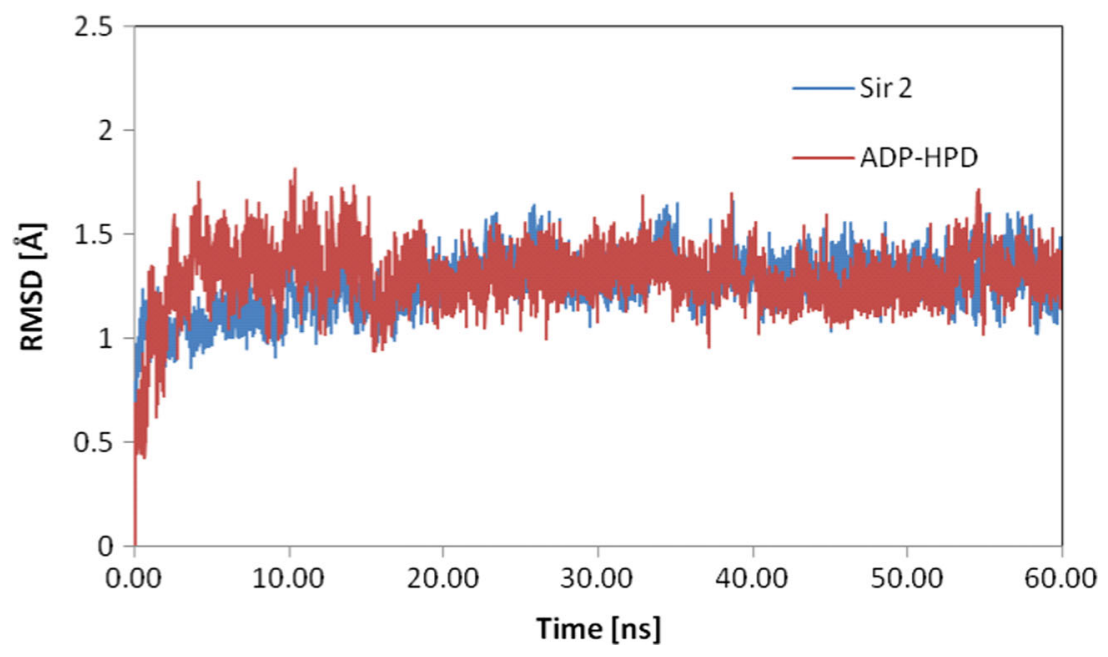

40 ns of trajectory, collected during molecular dynamics simulation, was used in analysis of the interaction between considered subunits. Structural analysis was performed with use of the VMD package [14]. In all molecular dynamics simulations, the AMBER 11 package was used [15].

\section{Intermolecular interaction energy partitioning}

Intermolecular interaction energy partitioning was performed for the protein-ADP-HPD complex resulting from molecular dynamics simulation. The analysis was performed for ADPHPD and amino acids forming a deacetylase binding pocket. In the present study, we discuss the results obtained using the variational-perturbational scheme implemented in a modified version of the GAMESS US package [16, 17$]$.

In this approach, the total intermolecular interaction energy calculated at Hartree-Fock level of theory $\left(\Delta \mathrm{E}^{\mathrm{HF}}\right)$ is decomposed into the first-order electrostatic $\left(\varepsilon_{\mathrm{el}}{ }^{(10)}\right)$, HeitlerLondon exchange $\left(\Delta \mathrm{E}_{\mathrm{ex}}^{\mathrm{HL}}\right)$ and delocalization components
$\left(\Delta \mathrm{E}_{\mathrm{del}}^{\mathrm{HF}}\right)$. The delocalization term, also referred to as the deformation component, contains charge-transfer and polarization effects. Since the dimer-centered basis set is employed during the calculations, both the total intermolecular interaction energy as well as its components are free from the basis set superposition error.

$\Delta E^{H F}=\varepsilon_{e l}^{[10]}+\Delta E_{e x}^{H L}+\Delta E_{d e l}^{H F}$

The analysis was performed for ADP-HPD and 15 amino acids forming a deacetylase binding pocket. The RHF/aug-ccpVDZ level of theory and about 1700 contracted Cartesian Gaussian basis functions were employed to describe the systems. Due to the size of the studied complexes, we have not attempted to determine interaction energy components resulting from the partition of the correlation energy at the MP2 level of theory (i.e., uncoupled dispersion energy, correlation correction to electrostatic energy and exchange-delocalization term) [18].
Table 1 Frequencies of hydrogen bond occurrence between ADP-HPD and amino acids from active site during molecular dynamics simulations

\begin{tabular}{|c|c|c|c|c|c|c|c|c|c|}
\hline & \multirow[t]{2}{*}{ Amino acids } & \multicolumn{8}{|c|}{ Hydrogen bond length $[\AA]$} \\
\hline & & 1.625 & 1.875 & 2.125 & 2.375 & 2.625 & 2.875 & 3.125 & 3.375 \\
\hline \multirow{10}{*}{$\begin{array}{l}\text { Frequency of } \\
\text { occurrence [\%] }\end{array}$} & ILE 117 & 0.5 & 15.0 & 32.7 & 27.2 & 14.4 & 7.2 & 2.1 & 0.7 \\
\hline & ASP 118(O1) & 9.1 & 35.2 & 6.3 & 0.6 & 1.3 & 3.0 & 5.5 & 7.8 \\
\hline & ASP $118(\mathrm{O} 2)$ & 9.0 & 32.8 & 5.7 & 1.2 & 2.8 & 8.1 & 12.1 & 12.7 \\
\hline & ALY 16 B & 13.6 & 19.6 & 6.2 & 1.7 & 1.7 & 1.5 & 1.3 & 1.4 \\
\hline & ASN 248 & 0.4 & 24.8 & 37.5 & 17.3 & 6.2 & 3.0 & 2.0 & 1.5 \\
\hline & SER 270 & 0.2 & 9.9 & 18.6 & 16.8 & 17.2 & 18.2 & 12.0 & 3.9 \\
\hline & LEU 249 & 0.1 & 11.8 & 34.2 & 26.6 & 13.4 & 7.5 & 2.8 & 1.7 \\
\hline & THR37 & 1.0 & 17.3 & 16.0 & 6.1 & 2.7 & 3.1 & 3.4 & 3.4 \\
\hline & ALA 33 & 0.8 & 25.7 & 34.4 & 15.2 & 5.2 & 1.5 & 0.5 & 0.4 \\
\hline & SER 225 & 0.0 & 0.0 & 0.0 & 0.7 & 5.2 & 17.1 & 26.5 & 22.7 \\
\hline
\end{tabular}

Values of hydrogen bond length represent middle values of intervals with a width of $0.25 \AA$ 
Fig. 4 Distribution of hydrogen bonds length created by oxygen atoms of ASP118 with ADP-HPD ligand

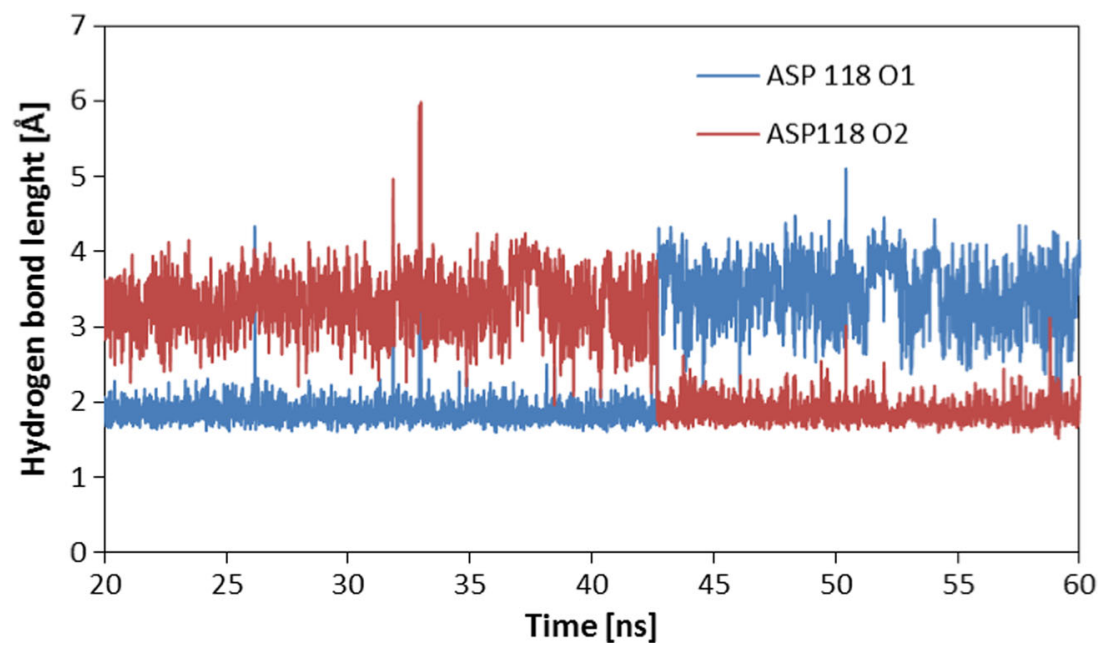

\section{Results and discussion}

\section{Docking and molecular dynamics simulations}

The docking procedure allows for a precise definition of structure of analyzed complex and interactions between both subunits. For all conformations obtained during the docking stage, the atoms of ADP-HPD ligand backbone are localized almost in this same points of conformational space of active site. Small differences are mainly related to localization of hydrogen atoms. The average value of binding affinity of the ligand obtained during this stage is $-12.15 \mathrm{kcal} / \mathrm{mol}$ and maximum differences between considered conformations do not exceed $0.1 \mathrm{kcal} / \mathrm{mol}$. An orientation of ADP-HPD in the enzyme active site determined at this stage exhibits a high similarity relative to experimental structure. Stability of this complex is maintained by numerous interactions among which hydrogen bonds play an important role. The carba-nicotinamide part of the ligand is stabilized by interactions created with ILE117, ASP118, and ALY16 B, adenine part of considered molecule creates bonds with ASN248, LEU249, THR37 and SER270, also oxygens from orthophosphoric part creates interactions with ALA33 and SER225. The trajectory obtained during $40 \mathrm{~ns}$ of equilibrated molecular dynamic simulation allowed evaluating the stability of the considered interactions. The values listed in Table 1 present distribution of hydrogen bond length for all conformations collected during the simulation. Bonds created by ADP-HPD with ILE117 and ASP118 are stable over all simulation times, in the case of bonds created by first amino acid interactions of medium and weak binding power dominate, while ASP118 creates mainly strong hydrogen bonds. Both oxygen atoms from rotatable carboxyl group play important roles in creation of interactions with ADP-HPD. The values presented in Fig. 4 unambiguously confirm that during whole simulation always one of oxygens atoms, from carboxyl group of aspartic acid, creates stable and strong hydrogen bond with hydrogen atom from nicotinamide part of ligand. Also, the adenine part of considered ligand creates stable hydrogen bonds. The most important interactions involve ASN248 and LEU249. In the case of first of mentioned amino acids, hydrogen bonds are present by $90 \%$ of simulation time, most of them is characterized by strong and medium binding power. Bindings created by LEU249 are observed in $95 \%$ of trajectory steps, their lengths indicate the dominant contribution of
Fig. 5 Distribution of distances between carbon atoms of phenylalanines and nicotinamide part of ADP-HPD ligand

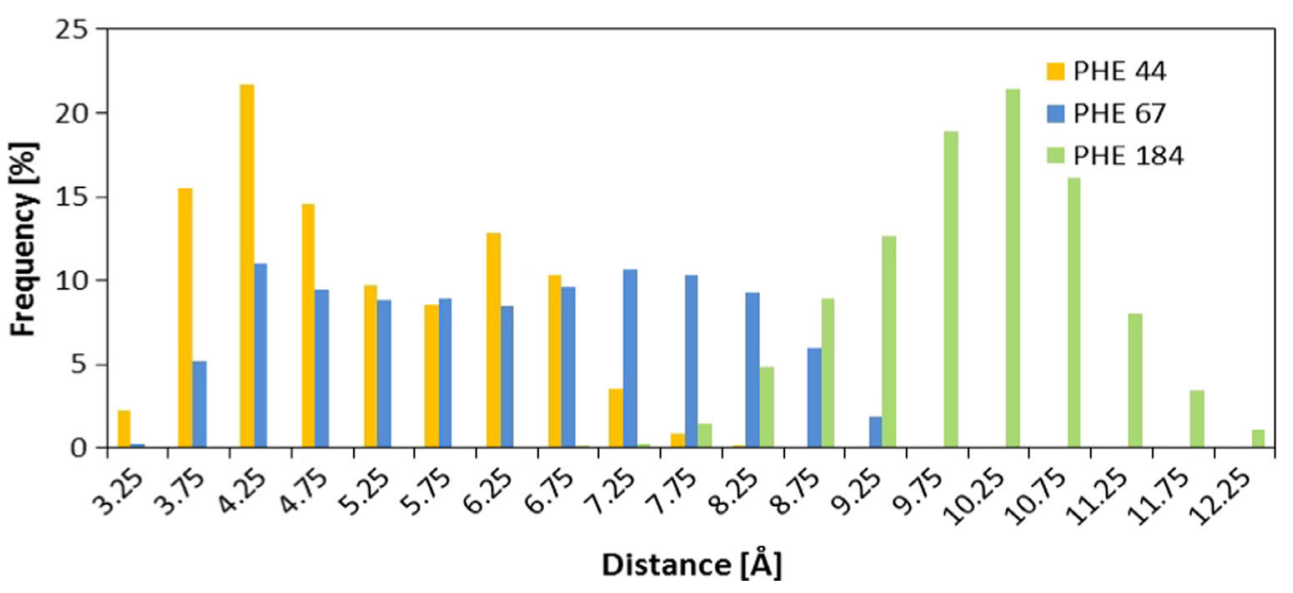


medium and weak interactions. The next non-negligible hydrogen bonds are created by SER 270 and THR 37 but in both cases frequency of occurring and binding power is much smaller than in the case of ASN248 and LEU249. The middle orthophosphoric part of ADP-HPD is stabilized by interactions, of strong and middle binding power, involving ALA33. Presented distribution of hydrogen bonds indicate on small mobility of ADPHPD in conformational space of active site what is confirmed also by stable RMSD values (av. $1.23 \pm 0.18$ ).

The next group of important forces related to stabilization of considered ligand is hydrophobic interactions. Active site of Sir 2 contains many amino acids, which could be involved in such interactions. The most important role can be fulfilled by aromatic amino acids localized in the vicinity of heterocyclic rings of ADP-HPD, namely phenyloalanies PHE44, PHE67, and PHE184. Figure 5 presents distances between aromatic systems of amino acids and nicotinamide part of considered ligand during molecular dynamics simulations. Presented distributions indicate that at least one of considered phenylalanine moieties plays an important role in stabilization of the ADP-HPD molecule. In the case of PHE44 by $50 \%$ of simulation time, the distance between aromatic rings does not exceed $5 \AA$, and mutual orientations between considered molecules allow the occurrence of stacking interactions. Also, localization of PHE67 in the active site during simulation ensures a large group of conformations, enabling the occurrence of hydrophobic interactions with heterocyclic ring of ADP-HPD.

Table 2 Intermolecular interaction energy partitioning

ADP-HPD-amino acid Intermolecular interaction energy components

\begin{tabular}{lrrrrl} 
& \multicolumn{1}{c}{$\mathrm{DE}^{\mathrm{HL}}$} & $\mathrm{e}_{\mathrm{el}}{ }^{(10)}$ & $\mathrm{DE}_{\mathrm{ex}}^{\mathrm{HL}}$ & $\mathrm{DE}_{\mathrm{del}}{ }^{\mathrm{HF}}$ & $\mathrm{DE}^{\mathrm{HF}}$ \\
\hline ARG 45 & -28.74 & -29.07 & 0.33 & -3.09 & -31.83 \\
ALA 33 & -5.90 & -18.02 & 12.12 & -10.51 & -16.41 \\
ASN 248 & 2.22 & -8.18 & 10.40 & -3.76 & -1.54 \\
GLY 34 & 0.08 & -5.09 & 5.17 & -2.63 & -2.55 \\
LEU 249 & 2.1 & -12.4 & 14.47 & -3.3 & $-1,2$ \\
PHE 184 & -0.80 & -1.20 & 0.39 & -0.66 & -1.46 \\
SER 270 & 3.74 & -9.408 & 13.15 & -4.34 & -0.60 \\
PHE 44 & 1.04 & -0.10 & 1.14 & -0.29 & 0.74 \\
PHE 67 & 0.66 & -0.44 & 1.10 & -0.22 & 0.44 \\
ALY 16 B & -4.15 & -15.64 & 11.48 & -5.15 & -9.30 \\
ILE 117 & 2.39 & -5.86 & 8.26 & -2.08 & 0.32 \\
THR 37 & 1.97 & -16.81 & 18.79 & -6.95 & -4.94 \\
SER 225 & 3.68 & -8.24 & 11.92 & -7.47 & -3.80 \\
ASP 118 & 13.59 & -9.85 & 23.44 & -12.68 & 0.90 \\
TYR 269 & 0.71 & -3.74 & 4.43 & -1.37 & -0.658 \\
ADP & -17.78 & -107.18 & 89.40 & -34.85 & -52.62 \\
NICO & 14.62 & -14.54 & 29.16 & -12.72 & 1.90 \\
\hline
\end{tabular}

The data are given in $\mathrm{kcal} / \mathrm{mol}$
Table 3 Spearman rank correlation coefficients estimated for intermolecular interaction energy components for considered pair

\begin{tabular}{lllll}
\hline & $\mathrm{e}_{\mathrm{el}}{ }^{(10)}$ & $\Delta \mathrm{E}_{\mathrm{ex}}{ }^{\mathrm{HL}}$ & $\Delta \mathrm{E}_{\mathrm{del}}{ }^{\mathrm{HF}}$ & $\Delta \mathrm{E}^{\mathrm{HF}}$ \\
\hline$\Delta \mathrm{E}^{\mathrm{HL}}$ & 0.14 & 0.6 & -0.3 & 0.6 \\
$\mathrm{e}_{\mathrm{el}}^{(10)}$ & & -0.5 & $\mathbf{0 . 7 6}$ & $\mathbf{0 . 7}$ \\
$\Delta \mathrm{E}_{\mathrm{ex}}^{\mathrm{HL}}$ & & & $\mathbf{- 0 . 8}$ & 0.025 \\
$\Delta \mathrm{E}_{\mathrm{del}}{ }^{\mathrm{HF}}$ & & & & 0.4 \\
\hline
\end{tabular}

\section{Selection criteria}

During this stage we selected the structure of the complex of ADP-HPD with Sir2 protein, which should be the most representative relative to the conformations occurring during considered simulation times. For this purpose, we used structural data describing distributions of 11 of the most important hydrogen bonds and distances between aromatic rings of the considered subunits. Several criteria were considered to choose a representative structure. One of the conditions that should fulfill the chosen structure was the smallest total value of considered hydrogen bonds, which should potentially correlate with the highest affinity of ligand toward active site. The second criteria required that hydrogen bond length and also distance between the considered aromatic subunits should belong to the most-represented intervals of distributions obtained during simulation times.

\section{Intermolecular interaction energy partitioning}

The existence of multiple hydrogen bonds between the ADPHPD molecule and the surrounding environment suggests that electrostatics should be important. Indeed, in terms of absolute numbers, its value is quite large, and has mainly a stabilizing character. Its highest value was found for the positively charged ARG45-ADP-HDP complex. As might be expected, the lowest values of electrostatic component (close to $0 \mathrm{kcal} /$ mol) were observed for complexes formed by the aromatic

Table 4 Properties of selected complexes

\begin{tabular}{llcl}
\hline Complex & Distance & Interaction energy & $\mathrm{DE}_{\mathrm{del}}^{\mathrm{HF} / \mathrm{e}_{\mathrm{el}}}{ }^{(10)}$ \\
\hline ILE 117 N-H...O & 2.03 & 0.32 & 0.35 \\
ALY 16 B O...H-O & 1.93 & -9.30 & 0.33 \\
ASN 248 N-H...O & 2.06 & -1.54 & 0.46 \\
SER 270 O-H...N & 2.13 & -0.60 & 0.46 \\
LEU 249 N-H...O & 1.94 & -1.2 & 0.27 \\
THR 37 O-H... & 1.82 & -4.94 & 0.41 \\
ALA 33 N-H...O & 2.01 & -16.41 & 0.58 \\
SER 225 N-H...O & 2.07 & -3.80 & 0.90
\end{tabular}

Distances $(\AA)$, intermolecular interaction energies $(\mathrm{kcal} / \mathrm{mol}), \mathrm{DE}_{\mathrm{del}}^{\mathrm{HF}} /$ $\mathrm{e}_{\mathrm{el}}{ }^{(10)}$ ratio 
ring of phenylalanine and ADP-HDP molecule. As is shown in Table 2, in the case of some complexes, the stabilization due to the mutual polarization of interacting subsystems reflected in the negative value of delocalization component (encompassing induction terms) and is also quite important. In the case of complexes containing ALA33 and ASP118, stabilization originates strongly from the interaction of permanent and induced multipole moments of subsystems due to their mutual polarization. In the case of phenylalanine and arginine complexes, the effects resulting from quantummechanical exchange of electrons between subsystems are particularly small. Some results indicate that although the first-order electrostatic is substantial, it might be completely quenched by associated Pauli repulsion. It was observed, for example, for weak interactions of ASN245 and TYR269 with ADP-HDP molecules. Consequently, it would be interesting to estimate the correlation contributions to the total intermolecular stabilization.

In order to characterize the relationships between intermolecular interaction energy components, Spearman rank correlation was used [19].

$\rho=1-6 \sum d_{i}^{2} / n\left(n^{2}-1\right)$

The data collected in Table 3 were obtained using the expression above, where $\mathbf{d}$ is the difference between ranks of the compared terms and $\mathbf{n}$ is the number of investigated complexes. It is seen that among of all pairs of components, the correlation coefficients of $\Delta \mathrm{Eel} / \Delta \mathrm{Edel}$ and $\Delta \mathrm{Eel} / \Delta \mathrm{EHF}$ are high. Between the exchange and delocalization components, an evident negative correlation with coefficient equal to -0.8 is observed (Table 4).

The nature of the hydrogen bonds stabilizing of investigated system can be characterized based on the theory of intermolecular interactions. Since the higher-order delocalization effects involve charge transfer excitation, they can be associated with covalent character of interactions. It was also shown by Grabowski et al. that delocalization has a dominant stabilizing character in the group of complexes with short dihydrogen bonds (distances close to $1 \AA$ ) [20]. As they observed for the remaining hydrogen bonds, electrostatic energy should be the most important. Based on the mentioned study, we can conclude that the hydrogen bonds that are partially covalent have an Edel/Eel ratio higher than 0.45 . In the case of our systems, consequently, the most covalent characters have ALA33 and SER225 complexes.

\section{Conclusions}

Each fragment of ADP-HPD ligand, namely nicotinamide, adenine nucleotides, and orthophosphoric chains, play important roles in stabilization of a complex with enzyme by numerous interactions of varying nature. The presented values describing time evolution of ADP-HPD molecule in the complex with Sir2 protein correlate with outcomes of docking procedure. Most all of the important interactions identified on the docking stage are present during molecular dynamics simulations. The greatest accumulation of interactions is observed in the space of D-pocket due to the presence of numerous hydrogen bonds (ILE117, ASP118, ALY16 B) and hydrophobic interactions (PHE44, PHE67), also in other regions of active site are important bindings present by most simulation time. Quite small and stable RMSD values of ADP-HPD molecule, supported by high frequency of bindings creation during whole simulation, indicate small mobility and limited conformational flexibility of considered ligands in the space of enzyme active site.

As is seen, even at the uncorrelated level of theory, the picture of interactions is quite complicated. Although in the case of the studied complex typical stacking motifs are not present between ADP-HPD molecules and neighboring amino acids, it follows from previous studies that one might expect non-negligible additional stabilization resulting from dispersion component of interaction energy [21]. The analysis of correlations between the intermolecular interaction components revealed existing dependence between positive and negative for $\Delta \mathrm{Eel} / \Delta \mathrm{Edel}$ and $\Delta \mathrm{Eex} / \Delta \mathrm{Edel}$, respectively.

Acknowledgments Calculations were performed at the Wroclaw Centre for Networking and Supercomputing.

Open Access This article is distributed under the terms of the Creative Commons Attribution 4.0 International License (http:// creativecommons.org/licenses/by/4.0/), which permits unrestricted use, distribution, and reproduction in any medium, provided you give appropriate credit to the original author(s) and the source, provide a link to the Creative Commons license, and indicate if changes were made.

\section{References}

1. Hunter CA (1993) Arene-arene interactions: electrostatic or charge transfer? Angew Chem Int Ed Engl 32:1584-1586

2. Gottlieb S, Esposito RE (1989) A new role for a yeast transcriptional silencer gene, SIR2, in regulation of recombination in ribosomal DNA. Cell 56:771-776

3. Bennett CB, Snipe JR, Westmoreland JW, Resnick MA (2001) SIR functions are required for the toleration of an unrepaired doublestrand break in a dispensable yeast chromosome. Mol Cell Biol 21: 5359-5373

4. Kaeberlein M, McVey M, Guarente L (1999) The SIR2/3/4 complex and SIR2 alone promote longevity in Saccharomyces cerevisiae by two different mechanisms. Genes Dev 13:2570-2580

5. Brachmann CB, Sherman JM, Devine SE, Cameron EE, Pillus L, Boeke JD (1995) The SIR2 gene family, conserved from bacteria to humans, functions in silencing, cell cycle progression, and chromosome stability. Genes Dev 9:2888-2902

6. Min J, Landry J, Sternglanz R, Xu RM (2001) Crystal structure of a SIR2 homolog-NAD complex. Cell 105:269-279 
7. Sanders BD, Zhao K, Slama JT, Marmorstein R (2007) Structural basis for nicotinamide inhibition and base exchange in Sir2 enzymes. Mol Cell 25:463-472

8. Zhao K, Harshaw R, Chai X, Marmorstein R (2004) Structural basis for nicotinamide cleavage and ADP-ribose transfer by $\mathrm{NAD}^{+}$-dependent Sir2 histone/protein deacetylases. Proc Natl Acad Sci U S A 101:8563-8568

9. Trott O, Olson AJ (2010) AutoDock Vina: improving the speed and accuracy of docking with a new scoring function, efficient optimization and multithreading. J Comput Chem 31(2):455-461

10. Bayly CI, Cieplak P, Cornell WD, Kollman PA (1993) A wellbehaved electrostatic potential based method using charge restraints for deriving atomic charges: the RESP model. J Phys Chem 97: 10269-10280

11. Jorgensen WL, Chandrasekhar J, Madura JD, Impey RW, Klein ML (1983) Comparison of simple potential functions for simulating liquid water. J Chem Phys 79:926-935

12. Adelman SA, Doll JD (1976) Generalized Langevin equation approach for atom/solid-surface scattering: general formulation for classical scattering off harmonic solids. J Chem Phys 64(6):2375-2388

13. Ryckaert JP, Ciccotti G, Berendsen HJC (1977) Numerical integration of the Cartesian equations of motion of a system with constraints: molecular dynamics of $n$-Alkanes. J Comput Phys 23(3): 327-341

14. HumphreyW DA, Schulten K (1996) VMD: visual molecular dynamics. J Mol Graph 14:33-38

15. Case DA, Darden TA, Cheatham TE III, Simmerling CL, Wang J, Duke RE, Luo R, Walker RCZW, Merz KM, Roberts B, Wang B,
Hayik S, Roitberg A, Seabra G, Kolossváry I, Wong KF, Paesani F, Vanicek J, Liu J, Wu X, Brozell SR, Steinbrecher T, Gohlke H, Cai Q, Ye X, Wang J, Hsieh MJ, Cui G, Roe DR, Mathews DH, Seetin MG, Sagui C, Babin V, Luchko T, Gusarov S, Kovalenko A, Kollman PA (2010) AMBER 11. University of California, San Francisco

16. Schmidt MW, Baldridgeidt MW, Baldridge KK, Boatz JA, Elbert ST, Gordon MS, Jensen JH, Koseki S, Matsunaga N, Nguyen KA, Su S, Windus TL, Dupuis M, Montgomery JAJ (2003) General atomic and molecular electronic structure system. Comput Chem $14: 1347-1363$

17. Gora RW (1998-2008) EDS package, Revision 2.8.1, Wrocław, Poland

18. Cybulski SM, Chałasinski G, Moszyński R (1990) On decomposition of second-order Möller-Plesset supermolecular interaction energy and basis set effects. J Chem Phys 92:4357-4363

19. Lehmann EL, D'Abrera HJM (1998) Nonparametrics: statistical methods based on ranks, rev. ed. Prentice-Hall, Englewood Cliffs, 292,300, and 323

20. Grabowski SJ, Sokalski WA, Jv L (2005) How short can the H...H intermolecular contact be? New findings that reveal the covalent nature of extremely strong interactions. J Phys Chem A 109(19): 4331-4341

21. Czyżnikowska Ż, Lipkowski P, Gora RW, Zaleśny R, Cheng AC (2009) On the nature of intermolecular interactions in nucleic acid base-amino acid side-chain complexes. J Phys Chem B 113:11511-11520 